\title{
Antifungal, optical, and mechanical properties of polymethylmethacrylate material incorporated with silanized zinc oxide nanoparticles
}

This article was published in the following Dove Press journal:

International Journal of Nanomedicine

27 March 2017

Number of times this article has been viewed

\author{
Krid Kamonkhantikul' \\ Mansuang Arksornnukit ${ }^{1}$ \\ Hidekazu Takahashi \\ 'Department of Prosthodontics, \\ Faculty of Dentistry, Chulalongkorn \\ University, Bangkok, Thailand; ${ }^{2}$ Oral \\ Biomaterials Engineering, Graduate \\ School of Medical and Dental \\ Sciences, Tokyo Medical and Dental \\ University, Tokyo, Japan
}

Background: Fungal infected denture, which is typically composed of polymethylmethacrylate (PMMA), is a common problem for a denture wearer, especially an elderly patient with limited manual dexterity. Therefore, increasing the antifungal effect of denture by incorporating surface modification nanoparticles into the PMMA, while retaining its mechanical properties, is of interest.

Aim of the study: This study aimed to evaluate antifungal, optical, and mechanical properties of heat-cured PMMA incorporated with different amounts of zinc oxide nanoparticles (ZnOnps) with or without methacryloxypropyltrimethoxysilane modification.

Materials and methods: Specimens made from heat-cured PMMA containing 1.25, 2.5, and $5 \%$ (w/w) nonsilanized (Nosi) or silanized ( $\mathrm{Si}$ ) ZnOnps were evaluated. Specimens without filler served as control. The fungal assay was performed placing a Candida albicans suspension on the PMMA surface for $2 \mathrm{~h}$, then Sabouraud Dextrose Broth was added, and growth after $24 \mathrm{~h}$ was determined by counting colony forming units on agar plates. A spectrophotometer was used to measure the color in L* (brightness), a* (red-green), b* (yellow-blue) and opacity of the experimental groups. Flexural strength and flexural modulus were determined using a three-point bending test on universal testing machine after $37^{\circ} \mathrm{C}$ water storage for $48 \mathrm{~h}$ and 1 month.

Results: The antifungal, optical, and mechanical properties of the PMMA incorporated with ZnOnps changed depending on the amount. With the same amount of ZnOnps, the silanized groups demonstrated a greater reduction in C. albicans compared with the Nosi groups. The color difference $(\Delta \mathrm{E})$ and opacity of the Nosi groups were greater compared with the Si groups. The flexural strength of the Si groups, except for the $1.25 \%$ group, was significantly greater compared with the Nosi groups.

Conclusion: PMMA incorporated with Si ZnOnps, particularly with 2.5\% Si ZnOnps, had a greater antifungal effect, less color differences, and opacity compared with Nosi ZnOnps, while retaining its mechanical properties.

Keywords: antifungal effect, optical properties, mechanical properties, silanized zinc oxide nanoparticles

\section{Introduction}

Correspondence: Mansuang Arksornnukit Department of Prosthodontics, Faculty of Dentistry, Chulalongkorn University, 34 Henri-Dunant Rd., Pathumwan, Bangkok 10330, Thailand

Tel +6622188532

Fax +6622188534

Email mansuang@yahoo.com
Denture is one tool of the dental armamentarium to rehabilitate patients with missing teeth. It enhances masticatory function, aesthetic, and phonetic properties and particularly improves the quality of life. The material mostly used for denture fabrication is polymethylmethacrylate (PMMA). However, PMMA can be reservoirs of microorganisms, which cause many oral diseases. Candida albicans is the most 
common causative pathogen of oral candidiasis, the disease associated with fungal infection. Denture-induced stomatitis is one form of candidiasis. ${ }^{1}$ Daily denture brushing is recommended to reduce the amount of microorganisms adhering to the denture surface. ${ }^{2}$ However, oral candidiasis can still occur in patients with predisposing factors, such as immunocompromised status, xerostromia, and age of the denture. ${ }^{1}$ Moreover, advancing age is also a risk factor for dentureinduced stomatitis in elderly patients due to their reduced cell-mediated immunity that may lead to fungal infection. The use of topical and/or systemic antifungal drugs may be inadequate in treating an oral fungal infection because the primary fungal reservoir in contaminated denture is not eliminated. Therefore, disinfection of the fungal reservoir within the denture should be performed. Several studies suggested methods of disinfection, such as microwave energy, ${ }^{3}$ ultrasonic soaking, ${ }^{4}$ and incorporation of metal $/$ metal oxide into PMMA. ${ }^{5-9}$ Silver nanoparticles (nps) are of interest because of their biocompatibility and antimicrobial effect., However, the incorporation of silver nps into PMMA did not improve the mechanical properties and caused discoloration of PMMA. ${ }^{5,9}$

Zinc oxide $(\mathrm{ZnO})$, a metal oxide, is widely used in dentistry as a restorative luting material, root canal filling material, and temporary restoration. This metal oxide varies in particle size, with sizes ranging from microns to nanometers. ${ }^{8,10,11}$ Many studies demonstrated the effectiveness of these particles on inhibiting the growth of fungi $i^{7,10}$ and bacteria. ${ }^{11-15}$ The inhibition depended on particle size, concentration, and type of microbes. Small particle size and high concentration of $\mathrm{ZnO}$ resulted in better antimicrobial activity. ${ }^{10,11}$

Studies on zinc oxide nanoparticles (ZnOnps) have demonstrated that different amounts of unmodified $\mathrm{ZnOnps}$ affected the mechanical properties of composite resin materials. ${ }^{13,14}$ A previous study investigated the effect of mixing methods of unmodified ZnOnps with methyl methacrylate in terms of particle size and distribution. ${ }^{8}$ Moreover, nanosized particles with a high surface energy resulted in aggregation of the particles. Particle aggregation generates weak points in the material. However, surface modification of these particles prevents the formation of aggregated nps. ${ }^{16,17}$

Silane coupling agents create a bond between organic and inorganic materials. These agents consist of two terminal functional groups; organofunctional groups, which establish bond with organic resin, and hydrolysable groups, which create bond with inorganic materials. A previous study found that the bond created by silane coupling agent was responsible for improving the mechanical properties of PMMA. ${ }^{18}$ To date, there has been no study about the mechanical properties of PMMA incorporated with surface-modified ZnOnps.

Many methods have been introduced to evaluate the color characteristics of a material. Visual measurement using shade tab in Munsell color system is convenient and low cost. ${ }^{19}$ However, this method is subjective and depends on various factors such as the mood and experience of the observer and illumination. A spectrophotometer is a reliable method to quantify the color characteristics of a material. The most commonly utilized color system for color assessment is the CIELAB system, which defines the color characteristics as $\mathrm{L}^{*}, \mathrm{a}^{*}$, and $\mathrm{b}^{*}{ }^{20-26} \mathrm{~L}^{*}$ represents brightness, $\mathrm{a}^{*}$ represents the red-green coordinates, and $b^{*}$ represents the yellow-blue coordinates. The CIELAB system can mathematically express the color difference $(\Delta \mathrm{E})$ between the two objects. Opacity is another optical property of a material, representing the ability of a material to block the passage of light. ${ }^{27}$ Opacity is based on the ratio of the reflectance of the material when backed by a black background compared with a white background.

Therefore, the objectives of this study were to evaluate antifungal, optical characteristics, and mechanical properties of PMMA incorporated with different amounts of ZnOnps with and without silanization. The null hypotheses were that 1) there would be no significant differences on the antifungal effect and optical characteristics among groups (PMMA incorporated with different amounts of $\mathrm{ZnOnps}$ with or without silanization) and 2) there would be no significant differences on the mechanical properties among groups (PMMA incorporated with different amounts of $\mathrm{ZnOnps}$ with or without silanization) and storage times (48 $\mathrm{h}$ and 1 month).

\section{Materials and methods Silanization of ZnOnps}

The materials used and their compositions are listed in Table 1 . The solution consisting of $2 \%(\mathrm{w} / \mathrm{w})$ of methacryloxypropyltrimethoxysilane, calculated from the weight of ZnOnps, and 70 volume\% ethanol was magneticallystirred for $30 \mathrm{~min}$. ZnOnps, without any purification, were dispersed into the solution and manually triturated until the solution was almost completely evaporated. The silanized (Si) ZnOnps were then left to dry at room temperature for 14 days before use.

\section{Specimen preparation}

Nonsilanized (Nosi) or ZnOnps of 1.25, 2.5, and 5\% (w/w), calculated from the weight of PMMA powder, were 
Table I Materials used and their compositions

\begin{tabular}{llll}
\hline Material/product name & Manufacturer & Batch number & Composition \\
\hline Zinc oxide nanoparticles/ZnOnps & $\begin{array}{l}\text { Nano Materials Technology } \\
\text { Co., Ltd. Chonburi, Thailand }\end{array}$ & $19041 \mathrm{I}$ & $\begin{array}{l}\text { Zinc oxide: (purity }>99.5 \%, \text { average particles } \\
\text { size 20-40 nm, specific surface area 26.22 } \mathrm{m}^{2} / \mathrm{g} \text { ) }\end{array}$ \\
$\begin{array}{l}\text { Heat-cured denture base material/SR } \\
\begin{array}{l}\text { Ivoclar Vivadent, Schaan, } \\
\text { Liechtenstein }\end{array}\end{array}$ & R84665 & $\begin{array}{l}\text { Powder: polymethylmethacrylate } \\
\text { Liquid: methyl methacrylate, ethylene glycol } \\
\text { dimethacrylate }\end{array}$ \\
$\begin{array}{l}\text { Silane coupling agent/ } \\
\text { 3-trimethoxysilyl propyl methacrylate }\end{array}$ & Sigma-Aldrich, St Louis, MO, & SHBD3265V & Methacryloxypropyltrimethoxysilane
\end{tabular}

homogeneously mixed with liquid monomer using a vortex mixer (VTX-3000L; LMS, Tokyo, Japan) for $10 \mathrm{~min}$. A mixture of pure PMMA powder and liquid monomer without ZnOnps served as the control group. Ninety-eight disc-shaped $(12 \times 2 \mathrm{~mm})$ specimens for the antifungal assay and optical characteristics test, and 112 bars $(64 \times 10 \times 3.3 \mathrm{~mm})$ for the mechanical properties test were fabricated. Polyvinylsiloxane material was injected into the molds to prepare silicone patterns. The patterns were invested in flasks with dental stone. After the dental stone set, the flasks were opened and the patterns were removed. The PMMA powder and liquid monomer were mixed at a powder/liquid ratio of $23.4 \mathrm{~g}: 10 \mathrm{~mL}$ and left until the mixture reached the dough stage. The mixture was then placed into the flask and hydraulic-pressed using a conventional method. The flasks were placed in a water bath that was heated up to $100^{\circ} \mathrm{C}$ and allowed to boil for $45 \mathrm{~min}$ to achieve polymerization according to the recommendations. After cooling to room temperature, the specimens were deflasked, the excess material was removed using a carbide bur in a low-speed rotary instrument, and wetpolished using 320 and 600-grit silicon carbide paper with a polishing machine (Nano 2000; Pace Technologies, Tucson, AZ, USA). The dimensions of the specimens were measured using a digital micrometer (minimum reading: $0.001 \mathrm{~mm}$, Digimatic Micrometer; Mitutoyo Corp., Kanagawa, Japan). The specimens were ultrasonically cleaned for $5 \mathrm{~min}$ to remove any debris and dried with compressed air.

\section{Antifungal assay}

C. albicans, ATCC 90028, was obtained as a stock culture from the Department of Microbiology, Faculty of Dentistry, Chulalongkorn University. The colonies were picked and transferred into a glass tube containing $3 \mathrm{~mL}$ of Sabouraud Dextrose Broth (SDB) (HiMedia Laboratories Pvt. Ltd., Mumbai, India) and incubated overnight at $37^{\circ} \mathrm{C}$. After incubation, the $C$. albicans suspension was transferred into new glass tube containing $3 \mathrm{~mL}$ of SDB and adjusted to achieve an optical density of $0.5-\mathrm{McF}$ arland suspension using a spectrophotometer (Nicolet Evolution ${ }^{\mathrm{TM}}$ 500; Thermo
Electron Corp., Madison, WI, USA) at $530 \mathrm{~nm}$. The suspension, consisting of approximately $1.5 \times 10^{6}$ cell $/ \mathrm{mL}$, was diluted 100 times and $100 \mu \mathrm{L}$ was spread on the Sabouraud Dextrose Agar (HiMedia Laboratories Pvt. Ltd.) to confirm the initial amount of $C$. albicans.

The fungal assay was modified from a previous study. ${ }^{9}$ The specimens were sterilized using low-temperature steam and formaldehyde. ${ }^{28}$ Disc-shaped specimens from each group were placed at the bottom of a 24-well cell culture plate (Costar, Corning, NY, USA). A $50 \mu \mathrm{L}$ C. albicans suspension was inoculated on the surface of the specimen in each well and left in the incubator with the lid slightly opened in a sterilized condition for $2 \mathrm{~h}$. After a $2 \mathrm{~h}$ incubation, each well was filled with $950 \mu \mathrm{L}$ of SDB. A mixture of the suspension and SDB served as the positive control while SDB alone served as the negative control. The 24-well culture plate was shaken using an orbital shaker placed in an incubator at $37^{\circ} \mathrm{C}$ for $24 \mathrm{~h}$. The colony-forming unit (CFU) of C. albicans in each well culture plate was determined using serial dilution method, followed by spreading on an agar plate. When determining the amount of $C$. albicans, only the agar plates with 20-200 CFU were counted. The amount of $C$. albicans was independently determined 6 times and averaged.

\section{Optical properties evaluation}

A spectrophotometer (Ultrascan XE; Hunter Associates Laboratory, Inc., Reston, VA, USA) was used to observe the differences in color as determined by the CIELAB system and opacity of disc-shaped specimens from CIEXYZ system using D65 illuminant and 10-degree observer. The specimens in each group $(n=8)$ were measured three times against the white and black background and averaged. L* (brightness), a* (redgreen coordinate), and $b^{*}$ (yellow-blue coordinate) of the CIELAB system were used to calculate the color differences of the specimens $(\Delta \mathrm{E})$, and Y (brightness) of the CIEXYZ system was used to evaluate the opacity of the specimens. The following equations were used: ${ }^{29}$

$$
\Delta \mathrm{E}=\left[\left(\mathrm{L}^{*}{ }_{1}-\mathrm{L}^{*}{ }_{2}\right)^{2}+\left(\mathrm{a}^{*}{ }_{1}-\mathrm{a}^{*}{ }_{2}\right)^{2}+\left(\mathrm{b}^{*}{ }_{1}-\mathrm{b}^{*}{ }_{2}\right)^{2}\right]^{1 / 2}
$$


where $\mathrm{L}^{*}{ }_{1}, \mathrm{a}^{*}{ }_{1}, \mathrm{~b}^{*}{ }_{1}=$ the mean value of the first group, $\mathrm{L}_{2}{ }_{2}$, $\mathrm{a}^{*}{ }_{2}, \mathrm{~b}_{2}^{*}=$ the mean value of the second group.

$$
\text { Opacity }=\left(\mathrm{Y}_{1} / \mathrm{Y}_{2}\right) \times 100
$$

where $Y_{1}=$ brightness of specimen backed by black background, $\mathrm{Y}_{2}=$ brightness of specimen backed by white background.

\section{Mechanical properties evaluation}

A three-point bending test was used to evaluate flexural strength and flexural modulus of the bar-shaped specimens according to ISO 20795-1. ${ }^{30}$ Sixteen specimens from each experimental group were randomly divided into two subgroups $(n=8)$ based on a storage time in $37^{\circ} \mathrm{C}$ deionized water for $48 \mathrm{~h}$ or 1 month before testing. A universal testing machine (EZ-SX; Shimadzu, Kyoto, Japan) was used at a crosshead speed of $5 \mathrm{~mm} / \mathrm{min}$ with a support span width of $50 \mathrm{~mm}$. Flexural strength and flexural modulus were calculated according to the following equations:

\section{Flexural strength $=3 \mathrm{FL} / 2 \mathrm{BH}^{2}$}

Flexural modulus $=\mathrm{F}_{1} \mathrm{~L}^{3} / 4 \mathrm{BH}^{3} \mathrm{D}$

where $\mathrm{F}=$ the maximum load $(\mathrm{N}), \mathrm{F}_{1}=$ the load at a point in straight line portion of the load/defection curve $(\mathrm{N})$, $\mathrm{L}=$ the support span width $(\mathrm{mm}), \mathrm{B}=$ the width of the specimen (mm), $H=$ the thickness of the specimen (mm), and $\mathrm{D}=$ the deflection at load $\mathrm{F}_{1}(\mathrm{~mm})$.

\section{Scanning electron microscope (SEM) observation and energy dispersive X-ray (EDX) analysis}

Fractured surfaces of the bar-shaped specimens of the control, 5Si, and 5Nosi groups after the three-point bending test were randomly selected, gold-sputter coated, and observed using SEM (Quanta 250; FEI Company, Eindhoven, the Netherlands) under acceleration of $20 \mathrm{kV}$ and magnification of $35,000 \times$.

One specimen was selected from the $5 \mathrm{Si}$ and 5 Nosi groups, carbon-sputter coated, and observed using the EDX detection system built into the SEM instrument (JSM5410LV; JEOL Ltd., Tokyo, Japan). Zn-mapping images of the surfaces were captured at an acceleration voltage of $20 \mathrm{kV}$ and a magnification of $2,000 \times$.

\section{Statistical analysis}

The $C$. albicans growth, $\mathrm{L}^{*}, \mathrm{a}^{*}, \mathrm{~b}^{*}$, and opacity data were analyzed using one-way analysis of variance (ANOVA)
$(P<0.05)$. The flexural strength and flexural modulus were analyzed by two-way ANOVA with groups (control, $1.25 \mathrm{Si}$, 1.25Nosi, 2.5 Si, 2.5Nosi, $5 \mathrm{Si}$, and $5 \mathrm{Nosi}$ ) and storage time (48 $\mathrm{h}$ and 1 month) as main factors $(P<0.05)$. Tukey Honestly Significant Difference's comparison test was used when homogeneity of variance was observed while Games-Howell comparison test was used when homogeneity of variance was rejected.

\section{Results}

One-way ANOVA of the C. albicans growth results demonstrated significant differences among experimental groups $(P<0.001)$. The Si groups showed a significant reduction in $C$. albicans compared with the control group, and in the Nosi groups, only the 5Nosi group showed a significant reduction (Figure 1). The reduction in C. albicans in the Si groups was greater than those of the Nosi groups with the same amount of ZnOnps.

One-way ANOVA of L*, $\mathrm{a}^{*}, \mathrm{~b}^{*}$, and opacity demonstrated significant differences among the experimental groups $(P<0.001)$ (Table 2). Photographs of the experimental groups demonstrated color change of the specimens as the amount of Nosi or Si ZnOnps increased (Figure 2). Increasing amounts of $\mathrm{ZnOnps}$ added into PMMA resulted in increased $\mathrm{L}^{*}, \Delta \mathrm{E}$, and opacity in both the Nosi and Si groups. In contrast, a greater decrease in $\mathrm{a}^{*}$ and $\mathrm{b}^{*}$ was observed as the amount of ZnOnps increased. $\Delta \mathrm{E}$ and opacity of the Nosi groups were greater than those of the Si groups containing the same amount of ZnOnps.

Two-way ANOVA of flexural strength data demonstrated significant differences only among the experimental groups ( $P<0.001$ ), while that of the flexural modulus results indicated significant differences on storage time $(P=0.015)$ and interactions $(P=0.006)$. Therefore, the flexural strength data of the two storage times were pooled and analyzed. Post hoc multiple comparison tests with mean and standard

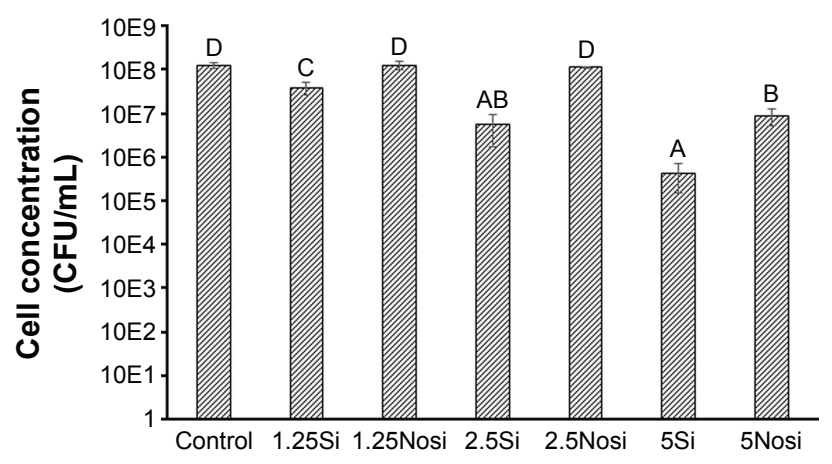

Figure I Mean and standard deviation of the growth of Candida albicans in each experimental group.

Note: Groups with the same letter $(A-D)$ were not significantly different $(P<0.05)$. Abbreviations: CFU, colony forming unit; Nosi, nonsilanized; Si, silanized. 
Table 2 Mean (standard deviation) of color characteristics of each experimental group

\begin{tabular}{llllll}
\hline Group & $\mathbf{L}^{*}$ & $\mathbf{a}^{*}$ & $\mathbf{b}^{*}$ & $\Delta \mathrm{E}$ & Opacity \\
\hline Control & $59.86(0.30)^{\mathrm{a}}$ & $21.48(0.24)^{\mathrm{g}}$ & $14.95(0.13)^{\mathrm{f}}$ & - & $71.3(1.0)^{\mathrm{a}}$ \\
I.25Si & $66.06(0.29)^{\mathrm{b}}$ & $20.55(0.28)^{\mathrm{f}}$ & $8.58(0.26)^{\mathrm{e}}$ & 8.9 & $87.0(0.8)^{\mathrm{b}}$ \\
I.25Nosi & $69.48(0.24)^{\mathrm{c}}$ & $19.77(0.14)^{\mathrm{e}}$ & $6.36(0.33)^{\mathrm{c}}$ & 13.0 & $91.6(0.6)^{\mathrm{c}}$ \\
$2.5 \mathrm{Si}$ & $70.04(0.34)^{\mathrm{d}}$ & $19.39(0.18)^{\mathrm{d}}$ & $7.33(0.17)^{\mathrm{d}}$ & 12.9 & $91.6(0.9)^{\mathrm{c}}$ \\
2.5 Nosi & $74.54(0.23)^{\mathrm{e}}$ & $17.25(0.14)^{\mathrm{c}}$ & $5.11(0.15)^{\mathrm{b}}$ & 18.2 & $95.8(0.5)^{\mathrm{d}}$ \\
$5 \mathrm{Si}$ & $75.42(0.30)^{\mathrm{f}}$ & $16.54(0.3 \mathrm{I})^{\mathrm{b}}$ & $5.30(0.22)^{\mathrm{b}}$ & 19.0 & $96.7(0.7)^{\mathrm{d}}$ \\
5 Nosi & $78.43(0.22)^{\mathrm{g}}$ & $14.94(0.09)^{\mathrm{a}}$ & $4.74(0.15)^{\mathrm{a}}$ & 22.2 & $98.0(0.3)^{\mathrm{e}}$
\end{tabular}

Notes: The different lowercase letters $(a-g)$ in the same column were significantly different $(P<0.05)$. '-' indicates no data.

Abbreviations: L*, brightness; $a^{*}$, red-green coordinates; $b^{*}$, yellow-blue coordinates; $\Delta \mathrm{E}$, color difference between corresponding group (Nosi or Si ZnOnps) and control group; Si, slianized; Nosi, nonsilanized.

deviation of flexural strength and flexural modulus are shown in Table 3.

After 1 month of water storage, the flexural strength and flexural modulus of the experimental groups did not significantly change. Compared with the control group, the flexural strengths of the $1.25 \mathrm{Si}, 1.25 \mathrm{Nosi}$, and $2.5 \mathrm{Si}$ groups were not significantly different, and the flexural moduli of the experimental groups were not significantly different at the same storage time. The flexural strength of the Si groups was significantly greater than those of the Nosi groups with the same amount of $\mathrm{ZnOnps}$ except for the $1.25 \%$ groups. Increased amount of $\mathrm{ZnOnps}$ resulted in increased reduction in the flexural strength; however, the flexural modulus did not show the same trend.

The fractured surface of the bar-shaped specimens of the control and 5\% groups were observed using SEM (Figure 3). The Si group image demonstrated well-distributed ZnOnps on its surface (Figure 3B), while that of the Nosi group showed aggregated $\mathrm{ZnOnps}$ on the fractured surface as indicated by white arrows in Figure 3C. The Zn-mapping images from EDX analysis demonstrated the distribution of $\mathrm{Zn}$ on the surface of the $5 \%$ groups (Figure 4 ). In the $5 \mathrm{Si}$ group, the $\mathrm{ZnOnps}$ were evenly dispersed (Figure 4A); however, in the 5Nosi group, the ZnOnps were densely aggregated in some areas (Figure 4B).

\section{Discussion}

The aim of the present study was to investigate the antifungal, optical, and mechanical properties of the incorporation with different amounts of Nosi or Si ZnOnps. Statistical analysis indicated that there were significant differences on antifungal effect and optical and mechanical properties among groups. Therefore, the null hypotheses were rejected.

This study found that increased amount of Si ZnOnps added into PMMA resulted in a significantly increased reduction in $C$. albicans, while among Nosi groups, a significant antifungal effect was only found in the 5Nosi group. Compared with the control group, the 5Si group demonstrated the highest reduction in C. albicans $(99 \%)$, followed by the $2.5 \mathrm{Si}$ group (95\%). The antifungal effect of the Si groups was greater than that of the Nosi groups at the same amount of $\mathrm{ZnOnps}$. The differences in antifungal effect between the Nosi and Si groups might be attributed to the distribution of $\mathrm{ZnOnps}$ in PMMA. It is likely that the Nosi nps aggregated due to their high surface energy into micron-scale particles, resulting in a low surface-to-volume ratio and so were poorly distributed in PMMA as shown in Figures 3C and 4B. In contrast, during silanization the hydroxyl groups of the $\mathrm{ZnOnps}$ react with the hydrolysable groups of the silane coupling agent, resulting in the silane coupling agent surrounding the $\mathrm{ZnO}$ particle, thus increasing

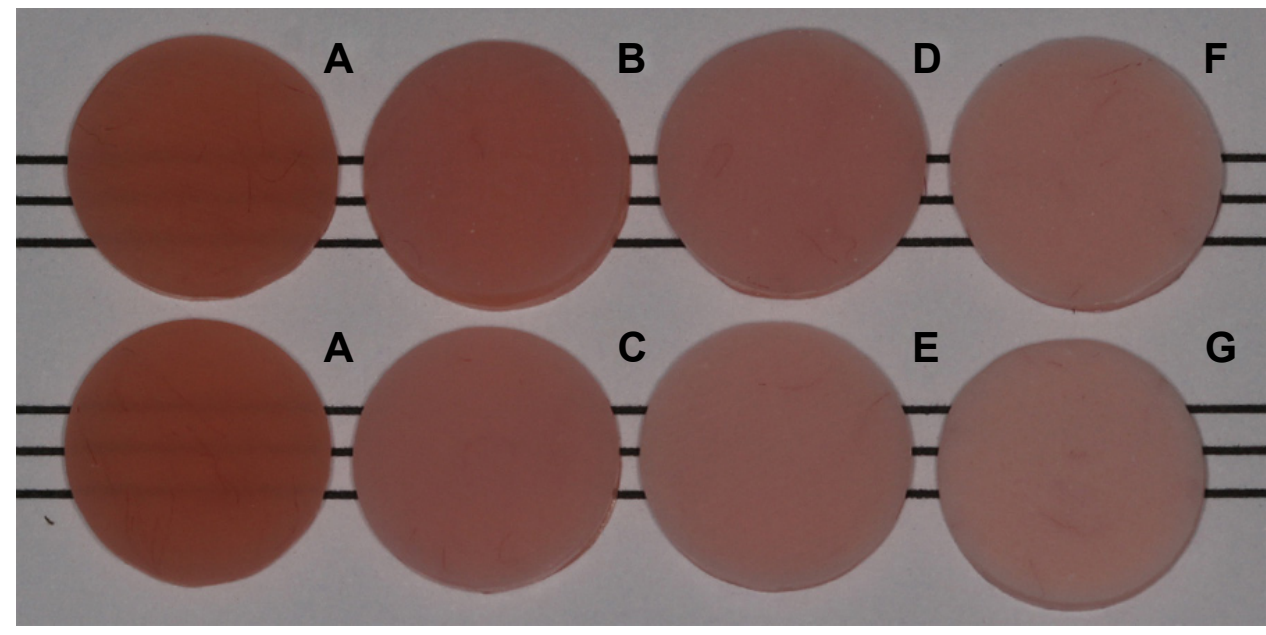

Figure 2 Photograph of the experimental groups: (A) control, (B) I.25Si, (C) I.25Nosi, (D) 2.5Si, (E) 2.5Nosi, (F) 5Si, and (G) $5 \mathrm{Nosi}$. Abbreviations: Nosi, nonsilanized; Si, silanized. 
Table 3 Mean (standard deviation) of flexural strength and flexural modulus of each experimental group

\begin{tabular}{llll}
\hline Group & $\begin{array}{l}\text { Flexural } \\
\text { strength }\end{array}$ & \multicolumn{2}{l}{ Flexural modulus } \\
\cline { 3 - 4 } & $92.92(5.08)^{\mathrm{c}}$ & $2.5 \mathrm{I}(0.05)^{\mathrm{a}, \mathrm{b}}$ & $\mathbf{I}$ month \\
\hline Control & $92.39(0.09)^{\mathrm{a}}$ \\
$\mathrm{I} .25 \mathrm{Si}$ & $92.85(3.26)^{\mathrm{c}}$ & $2.52(0.08)^{\mathrm{a}, \mathrm{b}}$ & $2.44(0.06)^{\mathrm{a}, \mathrm{b}}$ \\
$\mathrm{I} .25 \mathrm{Nosi}$ & $89.57(3.78)^{\mathrm{c}}$ & $2.48(0.06)^{\mathrm{a}, \mathrm{b}}$ & $2.41(0.06)^{\mathrm{a}}$ \\
$2.5 \mathrm{Si}$ & $89.8 \mathrm{I}(5.35)^{\mathrm{c}}$ & $2.43(0.13)^{\mathrm{a}, \mathrm{b}}$ & $2.48(0.07)^{\mathrm{a}, \mathrm{b}}$ \\
$2.5 \mathrm{Nosi}$ & $81.78(5.52)^{\mathrm{b}}$ & $2.50(0.04)^{\mathrm{a}, \mathrm{b}}$ & $2.47(0.06)^{\mathrm{a}, \mathrm{b}}$ \\
$5 \mathrm{Si}$ & $83.03(3.84)^{\mathrm{b}}$ & $2.49(0.1 \mathrm{I})^{\mathrm{a}, \mathrm{b}}$ & $2.5 \mathrm{I}(0.05)^{\mathrm{a}, \mathrm{b}}$ \\
5 Nosi & $69.13(5.79)^{\mathrm{a}}$ & $2.54(0.04)^{\mathrm{b}}$ & $2.44(0.06)^{\mathrm{a}, \mathrm{b}}$ \\
\hline
\end{tabular}

Note: The different lowercase letters $(a-c)$ in the same column were significantly different $(P<0.05)$.

Abbreviations: Nosi, nonsilanized; Si, silanized.

the distance among ZnOnps. Therefore, the Si ZnOnps will be well-distributed in PMMA and maintain their nanoparticle size, resulting in a high surface-to-volume ratio. ${ }^{10,17}$ This increased ratio led to a greater exposure area of ZnOnps on the surface in the Si groups compared with the Nosi groups. An increased contact area between the ZnOnps and C. albicans might contribute to the increased antifungal effect. The antifungal effect might also attribute to the dissociation of $\mathrm{Zn}$ ions from the ZnOnps. $\mathrm{Zn}$ ions can dissociate from the silane molecules because of the hydrolysable group of the silane coupling agent, which has a hydrophilic terminal. The hydrophilic terminal attracts water molecules and undergoes hydrolysis. This reaction may explain the finding that increased silane coupling agent in PMMA resulted in more water absorption. ${ }^{31}$ Moreover, the dissociation energy of the $\mathrm{Si}-\mathrm{O}$ bond in the silane coupling agent is higher than that of $\mathrm{Zn}-\mathrm{O} .^{32}$ Therefore, unbound $\mathrm{Zn}$ ions that have an antifungal effect can easily dissociate due to water absorbing into the material.

This study clearly shows that adding ZnOnps caused drastic color changes as also demonstrated in a previous study of silver nps. ${ }^{9}$ A previous study demonstrated that the threshold for detecting gingival color differences was $3.1 \pm 1.5 .{ }^{26} \Delta \mathrm{E}$ of all groups exceeded the threshold established for color acceptability in dentistry $(\Delta \mathrm{E}=2.7) .{ }^{25}$ Furthermore, $\Delta \mathrm{E}$ and opacity of the Nosi and Si groups increased as more amount of ZnOnps was added into PMMA. These changes in the optical characteristics of a material are a common disadvantage of incorporating metal oxide into PMMA. Therefore, the use of metal oxide should take into account the possible color change, especially in the aesthetic zone. However, the color of the gingiva of the denture can be clinically corrected by extrinsic coloration. Interestingly, it was found that adding ZnOnps resulted in increased $\mathrm{L}^{*}$, indicating that the material became brighter. Therefore, it may be possible to clinically correct the color by staining PMMA denture base material. ${ }^{33}$ Extrinsic coloration can be easily performed clinically to reduce the brightness of the material than with low-brightness material such as denture base incorporated with silver. Discoloration of silver particles due to oxidative reaction causes a metallic appearance of the denture base.

At the same amount of ZnOnps, the $\Delta \mathrm{E}$ and opacity of the Nosi groups was greater than those of the Si groups. This result might be explained by the even distribution of the Si ZnOnps. Aggregation of the Nosi ZnOnps resulted in almost completely blocking the transmission of light and a drastic color change. In contrast, silanization enhanced the distribution of the ZnOnps. Thus, the ZnOnps were homogeneously blended into the mixture between PMMA powder and liquid monomer, resulting in less color and opacity changes. Notably, $\Delta \mathrm{E}$ and opacity between the Nosi and Si groups decreased as the amount of ZnOnps increased. This result suggests that silanization has little effect on $\Delta \mathrm{E}$ and opacity when large amounts of ZnOnps were added.
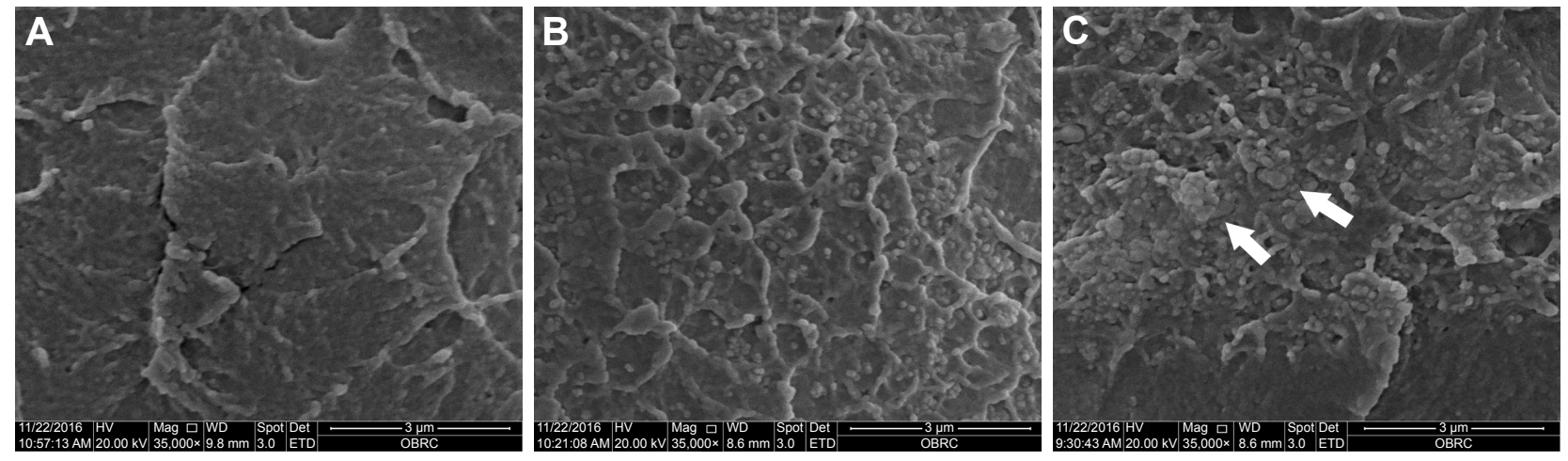

Figure 3 Representative SEM images of the fractured surface of specimen (35,000×): (A) control, (B) 5Si, and (C) $5 \mathrm{Nosi}$.

Note: The white arrows indicated the aggregated ZnOnps on the fractured surface of specimen.

Abbreviations: SEM, scanning electron microscope; Si, silanized; Nosi, nonsilanized. 

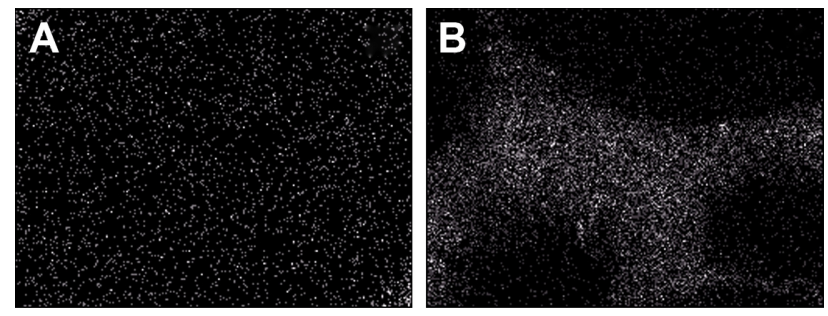

Figure 4 Representative Zn-mapping images of the surface of specimen using EDX on SEM (2,000×): (A) 5Si and (B) 5Nosi.

Abbreviations: EDX, energy dispersive X-ray; SEM, scanning electron microscope; Si, silanized; Nosi, nonsilanized.

The flexural strength and flexural modulus of the experimental groups after $48 \mathrm{~h}$ and 1 month of water storage were greater than $65 \mathrm{MPa}$ and $2 \mathrm{GPa}$, respectively, as required by ISO 20795-1. ${ }^{30}$ Flexural strength is influenced by various factors such as the size, shape, and amount of filler, and especially silanization. ${ }^{32,34}$ In the present study, flexural strength was ZnOnps amount-dependent, especially in the Nosi groups. The flexural strength of the Si groups was greater than that of the Nosi groups at the same amount of ZnOnps except for the $1.25 \%$ groups, which were not significantly different. This indicated that a small amount of ZnOnps did not affect the mechanical properties of PMMA. However, increased amount of ZnOnps resulted in more filler aggregations that were weak points in the specimen. Silanization enhanced the dispersion of ZnOnps in the liquid monomer and decreased particle aggregation. This may be why the flexural strength of the Si groups was higher compared with the Nosi groups. Furthermore, fillers play an important role in improving the mechanical properties of a material. As demonstrated in a previous study, whiskershaped aluminum borate filler was tailored to confront the breakage stress, improving the mechanical properties of PMMA. ${ }^{34}$ However, the addition of ZnOnps as filler in the present study did not result in an improvement in the mechanical properties of PMMA. This finding is likely due to the particles' spherical shape, which results in stress being evenly transferred through the resin matrix of the specimen. Moreover, $\mathrm{ZnO}$ is a soft filler, which allows stress to pass directly through the $\mathrm{ZnO}$ compared with other hard fillers such as silica or glass filler.

The flexural modulus of the Nosi and Si groups did not significantly change compared with the control group at each water storage time in this study. The flexural modulus was influenced by filler content. However, the 5\% ZnOnps added into PMMA in this study was considered a small amount. Therefore, it did not show significant effect on flexural modulus.
This research is the first to use $\mathrm{Si} \mathrm{ZnOnps}$ incorporated into PMMA to create the material that has an antifungal effect while maintaining its mechanical properties. It has also been demonstrated that the addition of ZnOnps in PMMA had a detrimental effect on the optical properties of PMMA. It was felt that the increased antifungal effect outweighs the color change because the color change can be adjusted using extrinsic coloration. However, future studies can determine the optimal concentration of ZnOnps incorporated into PMMA that results in a balance between the antifungal effect and the detrimental effect on opacity. Moreover, the addition of a secondary filler that has refractive index close to PMMA and harder than ZnOnps may help in reinforcing the mechanical properties without causing more color change. This aspect should also be investigated in the future studies. The results of this study suggest that $2.5 \mathrm{Si} \mathrm{ZnOnps}$ incorporated into PMMA might fulfill the requirement in terms of antifungal and mechanical properties. Although the color differences of the PMMA might be an aesthetic concern, this can be corrected by denture characterization using extrinsic coloration. Therefore, this nanocomposite material might be a material to inhibit the growth of $C$. albicans in the PMMA that causes denture-induced stomatitis. This material might be used as a treatment denture, a therapeutic denture used for a specific period, or for treating denture induced stomatitis in patients, especially in the elderly patient with limited manual dexterity. However, the long-term evaluation of the antifungal effect of PMMA denture base material incorporated with $\mathrm{Si} \mathrm{ZnOnps}$ both in vitro and in vivo is recommended.

\section{Conclusion}

Within the limitations of this study, it was concluded that PMMA incorporated with Si ZnOnps, particularly with $2.5 \%$ silanized ZnOnps, had a greater antifungal effect, less color differences, and less opacity compared with Nosi ZnOnps, while retaining its mechanical properties.

\section{Acknowledgment}

This work was supported by Faculty Research Grant (DRF57002), Faculty of Dentistry, Chulalongkorn University. The authors thank Dr Kevin Tompkins for language revision of this manuscript.

\section{Disclosure}

The authors report no conflicts of interest in this work.

\section{References}

1. Gleiznys A, Zdanavičienė E, Žilinskas J. Candida albicans importance to denture wearers. A literature review. Stomatologija. 2015;17(2): 54-66. 
2. Nishi Y, Seto K, Kamashita Y, Take C, Kurono A, Nagaoka E. Examination of denture-cleaning methods based on the quantity of microorganisms adhering to a denture. Gerodontology. 2012;29(2):e259-e266.

3. Senna PM, da Silva WJ, Cury AA. Denture disinfection by microwave energy: influence of Candida albicans biofilm. Gerodontology. 2012; 29(2):e186-e191.

4. Kawasaki K, Kamikawa Y, Sugihara K. In vitro and in vivo removal of oral Candida from the denture base. Gerodontology. 2016;33(2): 247-252.

5. Acosta-Torres LS, Mendieta I, Nuñez-Anita RE, Cajero-Juárez M, Castaño VM. Cytocompatible antifungal acrylic resin containing silver nanoparticles for dentures. Int J Nanomedicine. 2012;7:4777-4786.

6. Casemiro LA, Gomes Martins CH, Pires-de-Souza Fde C, Panzeri H. Antimicrobial and mechanical properties of acrylic resins with incorporated silver-zinc zeolite - part I. Gerodontology. 2008;25(3):187-194.

7. Cierech M, Kolenda A, Grudniak AM, et al. Significance of polymethylmethacrylate (PMMA) modification by zinc oxide nanoparticles for fungal biofilm formation. Int J Pharm. 2016;510(1):323-335.

8. Cierech M, Wojnarowicz J, Szmigiel D, et al. Preparation and characterization of $\mathrm{ZnO}-\mathrm{PMMA}$ resin nanocomposites for denture bases. Acta Bioeng Biomech. 2016;18(2):31-41.

9. Nam KY, Lee CH, Lee CJ. Antifungal and physical characteristics of modified denture base acrylic incorporated with silver nanoparticles. Gerodontology. 2012;29(2):e413-e419.

10. Lipovsky A, Nitzan Y, Gedanken A, Lubart R. Antifungal activity of $\mathrm{ZnO}$ nanoparticles - the role of ROS mediated cell injury. Nanotechnology. 2011;22(10):105101.

11. Padmavathy N, Vijayaraghavan R. Enhanced bioactivity of $\mathrm{ZnO}$ nanoparticles - an antimicrobial study. Sci Technol Adv Mater. 2008; 9(3):035004.

12. Aydin Sevinç B, Hanley L. Antibacterial activity of dental composites containing zinc oxide nanoparticles. J Biomed Mater Res B Appl Biomater. 2010;94(1):22-31.

13. Niu LN, Fang M, Jiao K, et al. Tetra-like zinc oxide whisker enhancement of resin composite. J Dent Res. 2010;89(7):746-750.

14. Tavassoli Hojati S, Alaghemand H, Hamze F, et al. Antibacterial, physical and mechanical properties of flowable resin composites containing zinc oxide nanoparticles. Dent Mater. 2013;29(5):495-505.

15. Tam KH, Djurišića $\mathrm{AB}$, Chan $\mathrm{CMN}$, et al. Antibacterial activity of $\mathrm{ZnO}$ nanorods prepared by a hydrothermal method. Thin Solid Films. 2008;516(18):6167-6174

16. Bressy C, Ngo VG, Ziarelli F, Margaillan A. New insights into the adsorption of 3-(trimethoxysilyl) propylmethacrylate on hydroxylated ZnO nanopowders. Langmuir. 2012;28(6):3290-3297.

17. Tang E, Cheng G, Ma X. Preparation of nano-ZnO/PMMA composite particles via grafting of the copolymer onto the surface of zinc oxide nanparticles. Powder Technol. 2006;161(3):209-214.
18. Chaijareenont P, Takahashi H, Nishiyama N, Arksornnukit M. Effect of different amounts of 3-methacryloxypropyltrimethoxysilane on the flexural properties and wear resistance of alumina reinforced PMMA. Dent Mater J. 2012;31(4):623-628.

19. Heydecke G, Schnitzer S, Türp JC. The color of human gingiva and mucosa: visual measurement and description of distribution. Clin Oral Investig. 2005;9(4):257-265.

20. Alghazali N, Burnside G, Moallem M, Smith P, Preston A, Jarad FD. Assessment of perceptibility and acceptability of color difference of denture teeth. J Dent. 2012;40 (Suppl 1):e10-e17.

21. Douglas RD, Steinhauer TJ, Wee AG. Intraoral determination of the tolerance of dentists for perceptibility and acceptability of shade mismatch. J Prosthet Dent. 2007;97(4):200-208.

22. Ho DK, Ghinea R, Herrera LJ, Angelov N, Paravina RD. Color range and color distribution of healthy human gingiva: a prospective clinical study. Sci Rep. 2015;5:18498.

23. Hyun HK, Kim S, Lee C, Shin TJ, Kim YJ. Colorimetric distribution of human attached gingiva and alveolar mucosa. J Prosthet Dent. 2017;117(2):294-302.

24. Ito M, Marx DB, Cheng AC, Wee AG. Proposed shade guide for attached gingiva - a pilot study. J Prosthodont. 2015;24(3):182-187.

25. Paravina RD, Ghinea R, Herrera LJ, et al. Color difference thresholds in dentistry. J Esthet Restor Dent. 2015;27 (Suppl 1):S1-S9.

26. Sailer I, Fehmer V, Ioannidis A, Hämmerle CH, Thoma DS. Threshold value for the perception of color changes of human gingiva. Int $J$ Periodontics Restorative Dent. 2014;34(6):757-762.

27. ISO/TR 28642. Dentistry - Guidance on Colour Measurement. Geneva, Switzerland: International Organization for Standardization; 2016.

28. Kanemitsu K, Kunishima H, Imasaka T, et al. Evaluation of a lowtemperature steam and formaldehyde sterilizer. J Hosp Infect. 2003; 55(1):47-52.

29. User manual. EasyMatch QC version 4.10 and Above User's Manual. Reston, VA: Hunter Associates Laboratory, Inc.; 2009.

30. ISO 20795-1. Dentistry-Base polymers-Part 1: Denture Base Polymers. Geneva, Switzerland: International Organization for Standardization; 2013.

31. Kanie T, Arikawa H, Fujii K, Inoue K. Physical and mechanical properties of PMMA resins containing gamma-methacryloxypropyltrimethoxysilane. J Oral Rehabil. 2004;31(2):166-171.

32. Dean JA. Lange's Handbook of Chemistry. 15th ed. New York, NY: McGraw-Hill, Inc.; 1999.

33. Berté JJ, Hansen CA. Custom tinting denture bases by visible light cure lamination. J Prosthodont. 1995;4(2):129-132.

34. Zhang X, Zhang X, Zhu B, Lin K, Chang J. Mechanical and thermal properties of denture PMMA reinforced with silanized aluminum borate whiskers. Dent Mater J. 2012;31(6):903-908.
International Journal of Nanomedicine

\section{Publish your work in this journal}

The International Journal of Nanomedicine is an international, peerreviewed journal focusing on the application of nanotechnology in diagnostics, therapeutics, and drug delivery systems throughout the biomedical field. This journal is indexed on PubMed Central, MedLine, CAS, SciSearch $®$, Current Contents ${ }^{\circledR} /$ Clinical Medicine,

\section{Dovepress}

Journal Citation Reports/Science Edition, EMBase, Scopus and the Elsevier Bibliographic databases. The manuscript management system is completely online and includes a very quick and fair peer-review system, which is all easy to use. Visit http://www.dovepress.com/ testimonials.php to read real quotes from published authors. 